\title{
Der perinatale Todesfall - Herausforderungen und Ressourcen
}

\author{
Der Tod eines Kindes kurz vor, während oder kurz nach der Geburt ist ein Schock für \\ die Eltern und immer auch eine Herausforderung für das betreuende Team. Hilfreiche \\ Ressourcen in dieser schwierigen Situation sind oft zu wenig bekannt.
}

Barbara Weiss-Egg

Korrespondenz:

Dr. med. Barbara Weiss-Egg Mooseggstrasse 13 CH-3550 Langnau

barbara.weiss-egg@gmx.ch

Auch Jahre nach dem Tod des Kindes empfinden Mütter und Väter noch tiefen Schmerz und Trauer um ihr verstorbenes Baby.
Man habe es weggebracht. Wohin? Wie hat es ausgesehen? War es ein Mädchen oder ein Knabe? Keine Antwort. Noch heute erinnert sich unsere Bekannte genau, wie sie damals vor rund 40 Jahren aus der Narkose erwacht ist. Bei der Einlieferung ins Spital damals im fünften Monat schwanger - hatte sie aufgrund der schockierenden Blutung bereits das Bewusstsein verloren. Und noch heute passiert es, dass sie in Tränen ausbricht, wenn das Thema unerwartet zur Sprache kommt. Kurz nach dem meist völlig unerwarteten Verlust des Kindes wurde die trauernde Mutter damals nach Hause geschickt, um das Geschehene möglichst rasch vergessen zu können. Man war wohl der Meinung, dass es die Trauer nur verstärken würde, wenn die Mutter ihr früh verstorbenes Kind zu Gesicht bekäme oder wenn man sie in den folgenden Tagen nochmals darauf anspräche.

Mein Mann und ich haben glücklicherweise ganz andere Erfahrungen gemacht, als wir vor nicht langer Zeit unsere Tochter aufgrund einer vorzeitigen Plazentalösung verloren haben. Mir wurde für die Notfallsektio eine Spinalanästhesie empfohlen, damit ich unser Kind, das aufgrund der Frühgeburtlichkeit keine grossen Chancen hatte, während der wenigen Minu-

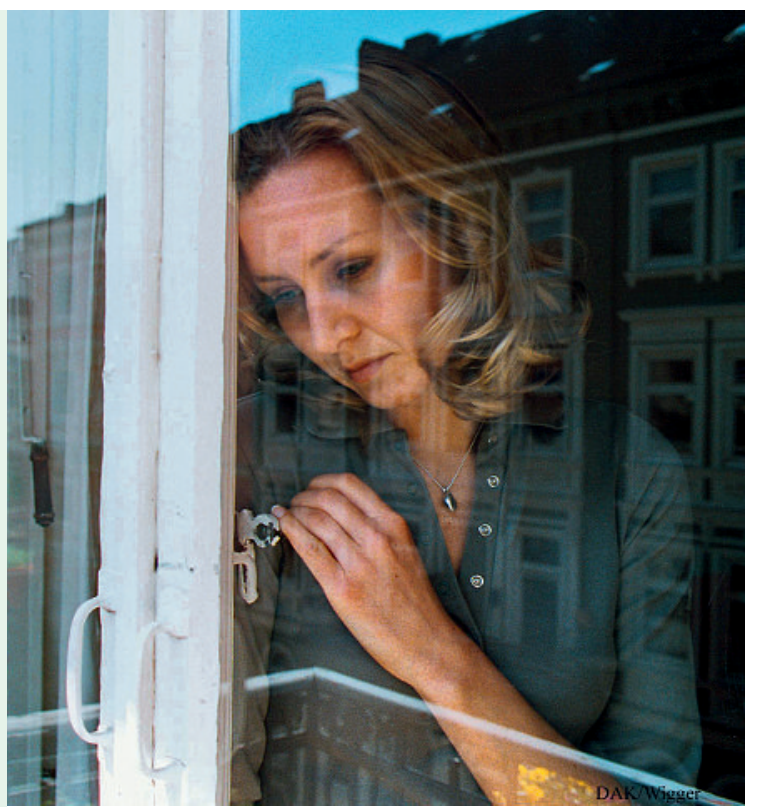

ten vor seinem Tod wach erleben konnte. Auch mein Mann wurde aufmerksam betreut und konnte während der Operation bei mir sein, sodass wir die kurze, aber intensive Zeit mit unserer Tochter gemeinsam durchlebten.

\section{Mutter ohne Kind}

Heute ist sich das Personal der Geburtshilfe-Abteilungen bewusst, dass Eltern auch nach dem Tod des Kindes Eltern bleiben. Sowohl körperlich als auch auf emotionaler Ebene wird eine Primipara durch den Verlust des Kindes nie mehr eine Nullipara. Da ein perinataler Todesfall praktisch immer völlig unerwartet eintritt, ist es entscheidend, den Eltern die Möglichkeit des bewussten Abschiednehmens zu geben. So haben wir es als hilfreich erlebt, unsere Tochter auch nach ihrem Tod noch einige Stunden in unserem Zimmer zu haben, sie anschauen und berühren zu können. In einer kürzlich publizierten Studie berichteten nahezu $80 \%$ der Eltern, die ihr peripartal verstorbenes Kind weder anschauten noch berührten, dass sie dies im Nachhinein bereuen [1]. Das Outcome der Mütter, die Kontakt zu ihrem Baby hatten, war bezüglich Sorgen und Depressivität besser als das derjenigen, die ihr verstorbenes Kind nicht gesehen hatten. Auch andere Verarbeitungshilfen werden je nach Spital umgesetzt; so wurden wir beispielsweise ermutigt, in einem eigens zu diesem Zweck geschaffenen Album (von den Pflegenden liebevoll «Sternenhimmel» genannt) eine Seite für unsere verstorbene Tochter zu gestalten. Die Berichte der anderen betroffenen Eltern zu lesen, hat uns sehr berührt.

Ebenso wichtig war für uns die Beerdigung. Wir entschieden uns für die Beisetzung in einem Gemeinschaftsgrab für früh verstorbene Kinder. Solche Grabstätten gibt es bereits in mehreren Kantonen, und vielerorts ist dort auch die Beisetzung von nicht-meldepflichtigen Kindern möglich [2].

\section{Wochenbett und Rückbildungskurs}

Trotz der sowohl medizinisch als auch menschlich gesehen hervorragenden Betreuung haben wir gemerkt, dass viele Ärzte wenig Kenntnisse über geltendes Recht und bestehende Angebote in dieser speziellen Situation haben. So ist vielen nicht bekannt, dass der be- 
zahlte Mutterschaftsurlaub nicht vom Weiterleben des Kindes abhängt und dass die ersten acht Wochen obligatorisch sind [3] (Voraussetzung für den Mutterschaftsurlaub ist eine Schwangerschaftsdauer von mindestens 23 vollendeten Wochen oder die Geburt eines lebensfähigen Kindes[4]).

Noch viel zu wenig bekannt ist die Tatsache, dass es bereits in mehreren Regionen der Schweiz Rückbildungskurse gibt, die spezifisch für Mütter nach Kindsverlust angeboten werden. Die Wochenbettbetreuung der betroffenen Frauen wird in der ohnehin sehr belasteten Situation tendentiell eher vernachlässigt [5]. Die «normale» Wöchnerin erhält automatisch Raum, sich sowohl mit ihrer Hebamme als auch mit anderen jungen Müttern über ihre Fragen und Sorgen auszutauschen. Nach perinatalem Kindsverlust wird die trauernde Mutter oft mit den Wochenbett-Themen, die über das Abstillen hinausgehen, verschont, was einerseits verständlich ist, andrerseits jedoch ein Manko an Information über normale Vorgänge des Körpers und entsprechende hilfreiche Massnahmen hinterlässt. Daher wird der Besuch eines Rückbildungskurses von den meisten Frauen als sehr wertvoll empfunden.

\section{Die Fachstelle}

Eine grosse Lücke schliesst die vor einigen Jahren gegründete Fachstelle Fehlgeburt und perinataler Kindstod (www.fpk.ch), die sowohl für Fachpersonen als auch für Betroffene hilfreiche Informationen und Angebote bereithält, so zum Beispiel Weiterbildungen für Personal im Bereich Neonatologie und Geburtshilfe. Auch ein Faltblatt existiert, das den Eltern in einer entsprechenden Situation abgegeben werden kann, um auf die vorhandenen Angebote hinzuweisen. Wenn

\section{Information für Betroffene \\ Faltblätter zum Themenkreis «perinataler Todes- fall» können angefordert werden unter:}

Fachstelle Fehlgeburt und perinataler Kindstod Postfach 480

CH-3000 Bern 25

Tel. 0313333360 (Mo-Fr 8.30-10.00 Uhr)

Fax 0313333362

fachstelle@fpk.ch / www.fpk.ch nur schon in jeder Geburtshilfe- und NeonatologieAbteilung sowie in den frauenärztlichen Praxen dieser Flyer vorhanden wäre und allen Betroffenen angeboten würde, wäre viel erreicht. Es ist wichtig, die Adresse einer kompetenten Anlaufstelle in den Händen zu haben, wenn man nach Spitalaustritt zu Hause ankommt und noch diverse Fragen offen sind. Praktische Tipps für Arbeitskollegen, Angehörige und andere indirekt Betroffene bietet das Merkblatt «Hilfreiches Verhalten im Umgang mit Eltern nach Kindsverlust», das von der Homepage heruntergeladen werden kann [6].

Auch mehrere Jahre nach dem Tod des Kindes empfindet ein Grossteil der Eltern noch tiefen Schmerz und Trauer um ihr verstorbenes Baby [7]. Es gibt jedoch hilfreiche Massnahmen, um für die betroffenen Mütter und Väter optimale Bedingungen zur Verarbeitung ihres Verlustes zu schaffen. Dieser Artikel soll dazu beitragen, die vorhandenen Ressourcen in der Ärzteschaft bekannt zu machen. Denn nicht nur Frauenärzte, auch Hausärzte, Psychiater, Kinderärzte und Vertreter anderer Fachrichtungen können mit dem Thema konfrontiert werden.

\section{Literatur}

1 Cacciatore J, Radestad I, Frøen F. Effects of Contact with Stillborn Babies on Maternal Anxiety and Depression. BIRTH 35:4 December 2008.

2 Jedes lebendgeborene Kind ist meldepflichtig, ebenso ein totgeborenes Kind, sofern es ein Geburtsgewicht von mindestens $500 \mathrm{~g}$ aufweist oder ein Gestationsalter von 22 vollendeten Schwangerschaftswochen erreicht.

3 Art. $35 a$ Abs. 3 ArG.

4 Art. 23 EOV.

5 Gassmann M, Maurer F. et al: Wochenbett bei Kindsverlust. Theoretische Grundlagen und praktische Ansätze für die Hebammenarbeit. Hebammenqualitätszirkel SHV Sektion Bern, Bern 2005. (erhältlich über www.fpk.ch).

6 www.fpk.ch $\rightarrow$ Betroffene Familien $\rightarrow$ Angebote $\rightarrow$ Selbsthilfe.

7 Büchi S, Mörgeli H, Schnyder U, Jenewein J, Hepp U, Jina E et al. Grief and Post-Traumatic Growth in Parents 2-6 Years after the Death of Their Extremely Premature Baby. Psychother Psychosom 2007; 76:106-114. 